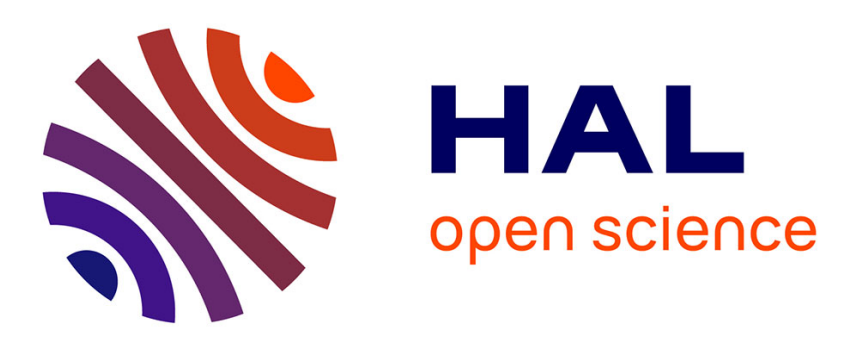

\title{
Processus identitaires dans la carrière des enseignants : deux études de cas en Éducation Physique et Sportive (EPS)
}

Thérèse Roux-Perez

\section{- To cite this version:}

Thérèse Roux-Perez. Processus identitaires dans la carrière des enseignants : deux études de cas en Éducation Physique et Sportive (EPS). STAPS : Revue internationale des sciences du sport et de l'éducation physique, 2006, 72 (2), 10.3917/sta.072.47 . hal-01716378

\section{HAL Id: hal-01716378 \\ https://hal.science/hal-01716378}

Submitted on 23 Feb 2018

HAL is a multi-disciplinary open access archive for the deposit and dissemination of scientific research documents, whether they are published or not. The documents may come from teaching and research institutions in France or abroad, or from public or private research centers.
L'archive ouverte pluridisciplinaire HAL, est destinée au dépôt et à la diffusion de documents scientifiques de niveau recherche, publiés ou non, émanant des établissements d'enseignement et de recherche français ou étrangers, des laboratoires publics ou privés. 


\section{PROCESSUS IDENTITAIRES DANS LA CARRIÈRE DES ENSEIGNANTS : DEUX ÉTUDES DE CAS EN ÉDUCATION PHYSIQUE ET SPORTIVE (EPS)}

Thérèse Roux-Perez

De Boeck Supérieur | «Staps »

2006/2 n 72 | pages 35 à 47

ISSN 0247-106X

ISBN 2804151859

Article disponible en ligne à l'adresse :

https://www.cairn.info/revue-staps-2006-2-page-35.htm

\section{Pour citer cet article :}

Thérèse Roux-Perez, " Processus identitaires dans la carrière des enseignants : deux études de cas en Éducation Physique et Sportive (EPS) », Staps 2006/2 (n $\left.{ }^{0} 72\right)$, p. 35-47.

DOI $10.3917 /$ sta.072.47

Distribution électronique Cairn.info pour De Boeck Supérieur.

(C) De Boeck Supérieur. Tous droits réservés pour tous pays.

La reproduction ou représentation de cet article, notamment par photocopie, n'est autorisée que dans les limites des conditions générales d'utilisation du site ou, le cas échéant, des conditions générales de la licence souscrite par votre établissement. Toute autre reproduction ou représentation, en tout ou partie, sous quelque forme et de quelque manière que ce soit, est interdite sauf accord préalable et écrit de l'éditeur, en dehors des cas prévus par la législation en vigueur en France. Il est précisé que son stockage dans une base de données est également interdit. 


\title{
Processus identitaires dans la carrière des enseignants : deux études de cas en Éducation Physique et Sportive (EPS)
}

\section{De Boeck Université | Staps}

\author{
2006/2 - no 72 \\ pages 35 à 47 \\ ISSN 0247-106X
}

Article disponible en ligne à l'adresse:

http://www.cairn.info/revue-staps-2006-2-page-35.htm

\section{Pour citer cet article :}

"Processus identitaires dans la carrière des enseignants : deux études de cas en Éducation Physique et Sportive (EPS)", Staps, 2006/2 no 72, p. 35-47. conditions générales d'utilisation du site ou, le cas échéant, des conditions générales de la licence souscrite par votre établissement. Toute autre reproduction ou représentation, en tout ou partie, sous quelque forme et de quelque manière que ce soit, est interdite sauf accord préalable et écrit de l'éditeur, en dehors des cas prévus par la législation en vigueur en France. II est précisé que son stockage dans une base de données est également interdit. 


\title{
Processus identitaires dans la carrière des enseignants : deux études de cas en Éducation Physique et Sportive (EPS)
}

\author{
THÉRÈSE ROUX-PEREZ
}

RÉsumé : L'étude s'intéresse à la carrière des enseignants d'EPS en repérant la manière dont ils appréhendent leur parcours professionnel, y trouvent des ancrages et développent des stratégies pour donner du sens à leur contexte de travail. Elle s'attache aux modalités d'appropriation des dispositifs de formation et à leur impact sur d'éventuels processus de transformation en termes d'identité professionnelle. Une enquête par questionnaire portant sur 381 sujets a été complétée par des entretiens semi directifs pour 12 d'entre eux. Les deux enseignants présentés dans cet article ont été choisis sur la base d'une entrée dans le métier faisant suite à des études très courtes, voire inexistantes, dans le domaine de l'EPS. L'un et l'autre ont obtenu, en cours de carrière, un concours de recrutement leur permettant d'accéder à un statut supérieur. Les résultats mettent en relief la diversité avec laquelle chaque enseignant, porteur d'une histoire singulière, investit son métier et se saisit des opportunités professionnelles ou personnelles qu'il rencontre. On remarque que l'obtention d'un diplôme de plus haut niveau ne produit pas toujours les effets escomptés par l'institution. Les trajectoires professionnelles s'organisent entre sentiment de compétence et espaces de reconnaissance sociale investis.

MoTS-CLÉS : identité professionnelle, carrière, représentations, formation.

\section{ABstract: Processes of change in teacher's professional identity: two case studies in Physical Education (PE)}

The investigation is interesting in Physical Education (PE) teacher's career. It is seen the way teachers apprehend their professional experiences, find prizes and unfold strategies give meaning to their work environment. It sticks to means appropriation teacher's training devices and their effect on possible process of change in professional identity. An investigation carried out through 381 questionnaires as been completed by semi-directive interviews for 12 of them. The two teachers described in this study has been choosed for first steps in profession after shorts or non existent PE teacher's training. Both have got, in process of carrer, a recruitment by competitive examination which enable to access to superior status. The findings highlight diversity which every teacher with his personal experience, get involved in profession and seize professionals and personals opportunities. We remark that gain of superior livel diploma don't always produce impacts that institution expect. The professionals experiences are got organized between competence consciousness and recognition area unfolded.

KEY WORDS: professional identity, career, conceptions, teacher training. 


\section{INTRODUCTION}

\subsection{Un contexte disciplinaire en mouvement}

Jusque dans les années 1980, les enseignants d'EPS du second degré ont pu accéder à leur profession par des examens ou concours correspondant à des niveaux de recrutement différents. Suite à des cursus de formation plus ou moins longs, l'entrée dans le métier s'effectuait sur la base de statuts hétérogènes ${ }^{1}$. Or, durant les vingt dernières années, l'EPS a vécu de profondes mutations institutionnelles dont les effets ont été ressentis au sein du groupe professionnel. En 1981, son entrée au ministère de l'Éducation nationale a confirmé un statut de discipline d'enseignement à part entière, inscrite entre continuités et changements : continuité des valeurs et des objectifs disciplinaires, changement dans le statut accordé à l'élève placé au centre des préoccupations éducatives ${ }^{2}$ (Roux-Perez, 2001). Dans ce contexte, les équipes disciplinaires ont été fortement sollicitées : construction de projets pédagogiques, traitement didactique des Activités Physiques Sportives et Artistiques (APSA), réflexion sur les contenus à enseigner et sur les modalités d'évaluation. Par ailleurs, de nouvelles procédures pédagogiques (différenciation, évaluation formative, pédagogie du contrat, mise en projet de l'élève, etc.) encouragées par l'institution, ont ouvert la voie à des transformations dans les pratiques. Parallèlement, des actions de formation continue et plus particulièrement des formations diplômantes ont été mises en place pour favoriser ce changement, l'hypothèse étant que le processus amorcé par la préparation des concours internes pourrait entraîner les acteurs concernés dans une dynamique d'innovation. Dans les faits, si nombre de professeurs d'EPS ont tenté de transformer leurs pratiques professionnelles, d'autres, formés sur des modèles antérieurs différents, se sont sentis en décalage avec cette évolution de la discipline, ignorant les nouveaux enjeux dont elle était l'objet. Ainsi, cette période s'est accompagnée d'un certain nombre de turbulences, voire de tensions au sein du groupe professionnel.

Un travail développé depuis plusieurs années sur les processus de construction identitaire nous a donc amenée à appréhender la carrière des enseignants d'EPS dans sa dimension à la fois objective (succession des positions occupées par l'enseignant au cours de sa vie) et subjective, c'est-à-dire en tant que processus de mise en cohérence par l'acteur de son cheminement professionnel (Hughes, 1937).

L'étude cherche en effet à comprendre la manière dont les enseignants, confrontés à une logique disciplinaire en mutation, investissent réellement leur parcours professionnel (Huberman, $1989^{3}$ ) et développent des stratégies pour donner du sens à leur environnement. Elle s'attache à repérer : a) les négociations, ajustements entre soi et les autres au sein et en dehors du contexte de travail ; b) le type de rapport établi entre pratique professionnelle et recommandations institutionnelles ; c) les modalités d'appropriation des dispositifs de formation et leur impact sur d'éventuels processus de transformation en termes d'identité professionnelle (Barbier, 1996 ; Dubar, 1991 ; Lautier, 2001).

\subsection{Des processus identitaires complexes dans la carrière des enseignants}

À l'échelle du groupe professionnel, l'identité, "c'est ce qui permet aux membres d'une même profession de se reconnaître eux-mêmes

1. Deux voies de formation initiale étaient envisageables après réussite à des tests physiques : un cursus de deux ans (post-Brevet) permettant, par la Maîtrise d'EPS, d'accéder au corps des Maîtres ; un autre de quatre ans (post-baccalauréat) permettant, par le CAPEPS (Certificat d'Aptitude au Professorat d'EPS), d'accéder au corps des certifiés.

2. Loi d'orientation sur l'éducation du 10 juillet 1989, Bulletin Officiel de l'Éducation Nationale spécial, n 4, 31 août 1989

3. L'étude s'intéresse au cycle de vie des enseignants et plus particulièrement à la période comprise entre 7 et 25 ans de carrière. M. Huberman (1989) caractérise cette période comme une phase de diversification (activisme) ou comme un moment de remise en question. 
et de faire reconnaître leur spécificité à l'extérieur » (Ion, 1990, 91). Sans renier ce premier niveau d'appartenance, on repère à l'intérieur du groupe des manières d'appréhender le métier sensiblement différentes : à la fois partagées par un certain nombre d'enseignants (Roux-Perez, 2003), elles sont aussi singulières, c'est-à-dire liées à l'histoire du sujet (modes de socialisation, réussites et échecs, projets, etc.), lui-même inscrit dans un contexte professionnel particulier. Ainsi, dans cette approche, l'identité personnelle est envisagée comme l'ensemble des représentations et des sentiments qu'une personne développe à propos d'elle-même, comme «ce qui permet de rester le même, de se réaliser soi-même et de devenir soi-même, dans une société et une culture donnée, et en relation avec les autres » (Tap, 1998, $65)$. De ce point de vue, en tant que processus complexe et dynamique, elle se situe à l'articulation de trois axes principaux plus ou moins en tension.

Tout d'abord, l'individu préserve le sentiment de rester le même au fil du temps et doit nécessairement s'adapter, en fonction de changements plus ou moins souhaités et/ou contrôlés : l'itinéraire professionnel intègre cet axe continuité-changement à travers un couplage entre histoire du sujet et modifications dans le contexte professionnel. Cela donne lieu à de multiples interprétations de la part des acteurs et à des formes de " transactions », pensées ou agies, qui traversent la situation individuelle et nécessitent délibérations, ajustements et compromis (Dubar, 1992).

Par ailleurs, l'individu élabore une image de soi en relation (accord, tension, contradiction) avec celles que, selon lui, les autres lui attribuent. Cela conduit à un sentiment de reconnaissance (des compétences, des diplômes, des savoirs acquis) ou de non-reconnaissance émanant de l'environnement humain, essentiel dans la construction identitaire. Mais si l'identité se fonde sur des actes d'attribution des autres, elle s'appuie aussi sur des revendications d'appartenances et de qualités, pour et par soimême. Ces autodéfinitions s'incarnent dans des rôles, des métiers mais intègrent paroles et attitudes des autres. Construites par chacun à travers les expériences de l'existence, elles résultent de la capacité du sujet à réagir, à rebondir face aux échecs, à trouver des stratégies pour s'adapter et poursuivre son projet identitaire (Dubar, 1998 ; Kaddouri, 2000).

Enfin, l'individu fait en sorte de conserver une unité (cohérence interne) tout en développant une relative diversité à travers de multiples facettes considérées comme autant de ressources sur lesquelles il peut prendre appui pour s'adapter aux changements et investir de façon plus ou moins dynamique la carrière dans laquelle il s'est engagé. Ainsi, le caractère subi ou volontaire du changement engage différemment le travail de recomposition de la personne vis-à-vis de la pluralité des mondes sociaux auxquels elle participe (Lahire, 1998).

L'identité professionnelle prend appui sur le « socle » de l'identité personnelle et y intègre des composantes professionnelles : pour trouver un équilibre, l'individu se représente la discipline, le métier, les pratiques professionnelles et s'engage dans l'action.

\subsection{Des représentations et des modes d'implication constitutifs d'une identité professionnelle}

Dans l'étude, les représentations sociales sont envisagées comme des formes de connaissances porteuses de valeurs qui donnent sens à la pratique et légitiment une vision du monde (Jodelet, 1989; Moscovici, 1961) ; elles orientent les conduites et amènent à privilégier certains types d'implication. Ainsi, l'individu s'investit en fonction des valeurs, des idéaux portés par son système de représentations et s'implique durablement dans le secteur qu'il s'est choisi, si cet engagement lui donne un sentiment de contrôle de la situation et une forme de reconnaissance sociale. En effet, l'engagement se traduit par des actes; il renvoie aux 
croyances, aux opinions qu'un individu défend personnellement, assume et revendique publiquement. En ce sens, s'engager revient à mettre en jeu une part importante de soi. Dans cette contribution, l'engagement est appréhendé comme « une démarche active d'appropriation et de partage de l'action menée, entraînant une implication durable» (Mias, 1998, 88). L'implication au travail est donc révélatrice des rapports que le sujet établit avec son environnement professionnel. De ce point de vue, prendre en considération les modes d'implication privilégiés par les enseignants à l'intérieur ou à l'extérieur du système scolaire, c'est tenter de comprendre ce qui fait sens pour eux, donne une cohérence à leur action et fonde, au moins en partie, leur identité. C'est aussi prendre en compte le «sens du travail», constitutif des identités professionnelles (Dubar, 2000) dans la mesure où il concerne à la fois le rapport à la situation de travail, l'engagement de soi dans l'activité et la reconnaissance de soi parmi les partenaires.

Ainsi, entre affirmation de soi et types de reconnaissance sociale ou institutionnelle, apparaissent des processus d'ajustement et de renégociation de l'identité. Déséquilibres, tensions, moments de crise sont dépassés si l'individu parvient à conserver la conscience de son unité, de sa continuité et une certaine maitrise des choix qu'il peut opérer à un moment donné de sa trajectoire personnelle et professionnelle. La perception de soi comme «agent, acteur ou auteur » (Ardoino, 1992) parait très liée à la façon dont chacun vit les situations ; elle renvoie au degré de contrôle ou de liberté que chacun pense avoir par rapport à celles-ci. Or, "plus un individu s'implique, plus il satisfait à son propre plaisir et à la consolidation de son identité professionnelle» (Mias, 1998, 143).

De ce point de vue, l'étude prend en compte le système «représentations-valeurs- pratiques » pour appréhender le rapport au métier (Charlot, 1997 ; Altet, 2000). Les représentations sont alors envisagées comme des systèmes de relation, d'interprétation et ancrées dans un réseau de significations. Pour s'adapter à des changements survenus durant la carrière et à l'évolution d'une discipline scolaire dans laquelle il se reconnaît plus ou moins, l'enseignant prend appui sur ce système et procède à des ajustements lui permettant de construire un monde cohérent et de se justifier de ses (dés)engagements.

\section{MÉTHOdOLOGIE}

Dans un premier temps, une approche par questionnaire (1998) portant sur 381 enseignants d'EPS de trois académies différentes a permis de repérer des profils distincts au niveau des trajectoires professionnelles (RouxPerez, 2003). Des entretiens semi-directifs ont ensuite été proposés à 12 sujets en 2000 . Une analyse structurale du discours, inspirée des travaux de Demazière et Dubar (1997), a été réalisée à partir :

a) des séquences ou épisodes du récit qui éclairent le parcours professionnel et en soulignent la dynamique ;

b) des « actants » qui interviennent dans le récit et avec lesquels s'établissent des systèmes de relations ;

c) des propositions ${ }^{4}$ ou arguments qui rendent compte des représentations à l'œuvre dans les discours et sont destinés à convaincre l'interlocuteur, à défendre son point de vue (valeurs, identité pour soi et pour autrui, rapport à l'institution). La méthode choisie, inductive, s'appuie donc sur l'interprétation de données langagières. Elle part du principe que tout locuteur construit, dans l'activité de mise en mots, une «structure d'univers simple organisée autour de quelques notions clés» (Ghiglione,

4. Ces propositions sont retranscrites entre guillemets et en italique dans la partie concernant les résultats de l'enquête. 
1991, 36). L'analyse s'attache à reconstruire la structure des oppositions dans le discours des enseignants, qui constitue en quelque sorte un programme argumentatif assurant au sujet une cohérence cognitive.

Dans cette contribution, deux enseignants ont été choisis :

a) sur la base d'une entrée dans le métier faisant suite à des études très courtes, voire inexistantes dans le domaine de l'EPS ;

b) pour avoir réussi la même année un concours de recrutement interne (CAPEPS) leur permettant d'accéder, en tant que professeur certifié d'EPS, à un statut supérieur.

Ces deux cas très contrastés invitent à repérer le poids effectif de la formation diplômante dans une carrière en termes d'effets sur les représentations et les pratiques des enseignants. Ils mettent en relief le jeu de tensions et de transactions qui en résultent entre :

a) continuité et changement : l'analyse porte sur le rapport à l'histoire sportive et scolaire intégrant une relation aux savoirs et à l'évolution disciplinaire. Elle montre dans quelle mesure le changement de statut obtenu par les deux enseignants de l'étude favorise réellement l'inscription dans une dynamique ;

b) soi et autrui : il s'agit de repérer les éventuels écarts entre définition de soi et reconnaissance d'autrui durant la carrière ;

c) unité et diversité: ce niveau prend en compte la diversité des facettes de l'individu, les espaces sociaux investis et la quête de cohérence que chacun tente d'établir entre ses différents registres de pensée et d'action.

Cet ensemble de tensions trouve son dépassement dans la construction d'un « monde ${ }^{5}$ permettant au sujet de trouver, momentanément au moins, une forme d'équilibre.

\section{RÉSULTATS}

\subsection{Présentation des sujets}

\subsubsection{Max ou "l'enseignant clivé »}

Âgé de 45 ans et titulaire du baccalauréat, Max vit une formation initiale difficile. Suite à plusieurs échecs qui ont compromis un accès au professorat, il réussit à être Maître d'EPS après une formation en deux ans jugée dévalorisante car accessible dès l'obtention du Brevet en fin de collège. Il enseigne alors pendant 20 ans dans un collège classé en ZEP, période durant laquelle il réussit le concours interne du CAPEPS. Au moment de l'enquête, il a obtenu depuis deux ans une mutation dans un lycée rural. Ancien sportif de niveau national en escrime, passionné par ce sport "tellement complet qu'il est inclassable ", il reste convaincu d'avoir sacrifié sa carrière sportive pour des études qui ne l'ont pas satisfait. Dès sa première affectation, il décide de créer un club dans sa discipline de prédilection. Très investi dans cette structure, il prolonge d'une autre manière sa réussite en ce domaine. Ainsi, le choix de l'enseignement s'est fait par défaut, même s'il a été perçu dans l'entourage familial comme une réelle ascension sociale.

Impliqué dans de multiples tâches au sein de son collège durant les premières années (coordonnateur en EPS, professeur principal, élu au conseil d'administration), il est entré en conflit avec certains collègues de travail qu'il accuse d'avoir «adhéré aux nouvelles orientations de l'EPS » à des fins carriéristes, tout en rejetant ceux qui, comme lui «ne rentraient pas dans le moule». Actuellement, Max fait preuve d'un engagement très relatif dans le métier d'enseignant et énonce un certain nombre de représentations négatives sur la discipline et sur l'institution.

5. Le terme de monde est envisagé ici comme une totalité d'appartenance, désignant un ensemble culturel spécifique porteur de significations. Il vise non pas la simple somme (d'objets, de propriétés, de comportements) mais un ordre de co-appartenance. Il exprime donc un mode de compréhension unificateur, un noyau de significations commun. 
De ce point de vue, il justifie ses pratiques en optant pour des «objectifs mesurés " avec des élèves qui sont "d'abord à rééduquer ", tout en faisant «le choix de l'efficacité ». Bien faire son métier pour un enseignant d'EPS, consiste à maîtriser les APSA, essentiellement d'un point de vue pratique. En ce sens, il réfute recommandations officielles et « théories didactiques » qu'il juge inopérantes, critique une formation initiale et continue des enseignants insuffisamment concrète, et, plus largement, construit un rapport négatif à l'institution : inspecteurs incompétents, dans une discipline soumise à des effets de mode (didactique) qui ne reconnaît plus les valeurs du sport.

Dans cet univers conflictuel, Max se définit comme un enseignant atypique qui sait mettre en avant sa mission d'éducation, quels que soient son domaine d'intervention et son public. Pour autant, et malgré sa réussite au CAPEPS qui constitue une reconnaissance institutionnelle, le regard porté par autrui semble quelque peu dissonant : perçu « comme un fumiste » par ses (ex-) collègues du collège, il se dit à présent reconnu au lycée car «pas prétentieux » et restant «à sa place dans une discipline somme toute secondaire ». En revanche, la gratification semble venir du club d'escrime, pensé « comme une petite entreprise » dans laquelle il est «à la fois directeur, professeur, gestionnaire ». Son fort investissement dans ce domaine l'a conduit à opter pour un travail à mi-temps au lycée.

\subsubsection{Michèle ou «l'enseignante intégrée »}

Âgée de 49 ans, titulaire du baccalauréat, Michèle commence sa carrière en tant qu'institutrice, conservant intact son désir initial d'être enseignante d'EPS. En effet, dès la classe de seconde, très intéressée par la pratique des activités physiques, motivée par des enseignants d'EPS qui lui «donnent une image positive du métier », elle tente le concours pour la formation de Maitre d'EPS. Elle échoue et poursuit donc ses études au lycée. Blessée lors des épreuves d'entrée au professorat, elle choisit d'enseigner en primaire, encouragée par ses parents, eux-mêmes instituteurs. Après avoir mené des études universitaires en Lettres, elle devient «PEGC Lettres-EPS ». Au moment de l'enquête, elle travaille depuis 20 ans dans un collège rural, période durant laquelle elle réussit le concours interne du CAPEPS. Parallèlement, elle pratique toujours de façon assidue le volley-ball, sport dans lequel elle a joué en compétition au niveau national et qu'elle investit actuellement en loisir.

Impliquée dans de multiples tâches au sein de l'établissement et en dehors, elle met en avant, dans le métier, des valeurs de justice et de respect. Visant la réussite de tous ses élèves, elle tient à adapter les contenus proposés, sans perdre de vue les objectifs disciplinaires. En ce sens, les textes officiels sont appréhendés comme un point d'appui, relayés par des actions de formation continue qu'elle suit avec intérêt. N'ayant pu bénéficier d'une formation initiale, elle déplore certaines lacunes dans la connaissance des APSA qu'elle comble en partie par des lectures et une recherche personnelle. L'inspection, vécue comme valorisante lors de sa titularisation, lui a donné l'occasion de prendre pleinement confiance dans une discipline qu'elle illustre de quelques mots : «éducation, travail des élèves, réflexion de l'enseignant », sans pour autant passer sous silence les « difficultés à tenir le cap » devant des élèves qu'il faut enrôler dans le travail, souvent recadrer, tout en veillant à accompagner au plan psychologique ceux qui en ont besoin.

Michèle se définit comme «centrée sur les apprentissages, à l'écoute mais exigeante ", et pense être perçue par les autres acteurs, dans et hors de l'école, comme quelqu'un de « courageux, dynamique et volontaire ». C'est en ce sens que l'entrée dans le métier d'enseignant d'EPS par un détour de plusieurs années est vécue comme une réussite tant au plan professionnel que personnel. Pourtant, des tensions existent dans un milieu où tous ne la reconnaissent pas comme une enseignante d'EPS à part entière et où sa position vis-à-vis de la discipline 
peut être perçue par le groupe professionnel comme trop proche de celle des inspecteurs.

Ainsi, pour dépasser certaines dissonances et rester en cohérence avec lui-même, chaque enseignant opère de multiples transactions avec son environnement institutionnel et humain et tente de justifier ses prises de position. Par ailleurs, l'analyse montre dans quelle mesure chacun parvient à réduire les tensions entre continuité et changement, soi-autrui, unitédiversité, pour construire en quelque sorte un «monde qui tient» (Derouet, 1992) et s'inscrire dans un processus identitaire gérable sur la durée de la carrière.

\subsection{La construction d'un monde cohérent}

\subsubsection{Le monde de Max}

3.2.1.1. Résoudre les tensions entre continuité et changement : une question de dosage

Très impliqué au sein d'un club qu'il a créé, Max se désengage de son métier d'enseignant qu'il dit assumer « a minima ». À travers ce choix de vie, il prolonge une réussite dans une voie qui a toujours été valorisante pour lui. Afin de garder une cohérence cognitive et une image de soi positive, il est amené à énoncer un certain nombre de représentations sur le métier, les élèves, les enseignants et l'institution dans son ensemble. Cette position semble paradoxale dans la mesure où la réussite du CAPEPS interne pourrait laisser croire à des changements professionnels. Il n'en est rien car cette reconnaissance institutionnelle questionne ses engagements personnels. De manière à rester équilibré, il rejette les éléments qui le mettraient en difficulté, envisageant cette réussite comme une opportunité mais surtout comme un dû de la part de l'État qui l'emploie. En ce sens, il se définit comme «quelqu'un de non prétentieux, non carriériste, qui a su rester le même au fil du temps » et continue à défendre un ancrage quasi exclusif sur les pratiques. Il peut ainsi trouver des raisons à son désengagement de l'enseignement de l'EPS : cette discipline devient trop intellectuelle et se prend trop au sérieux. De façon récurrente, son discours renvoie à une forte opposition EPS/club, doublée d'un autre clivage très important en termes identitaires: il se perçoit comme un «agent» de l'Éducation nationale qui remplit simplement les différentes tâches qui lui sont assignées et comme un réel "auteur " au sein de son club, entièrement responsable des choix qu'il est amené à faire (Ardoino, 1992).

Tableau 1. Structure des oppositions dans le discours de Max

\begin{tabular}{|c|c|c|c|}
\hline \multirow[t]{2}{*}{ Intérêt pour le club (+) } & \multicolumn{2}{|c|}{ Termes associés dans le discours } & \multirow[t]{2}{*}{ Désintérêt pour l'EPS (-) } \\
\hline & avec le club & avec l'EPS & \\
\hline $\begin{array}{c}\hat{\text { Etre } ~} \text { auteur": } \\
\text { "fonctionner comme un chef d'entreprise" }\end{array}$ & $\begin{array}{c}\text { concret } \\
\text { pratique } \\
\text { fond: efficacité } \\
\text { physique } \\
\text { pas d'obligation } \\
\text { liberté }\end{array}$ & $\begin{array}{c}\text { abstrait } \\
\text { théorie } \\
\text { forme : discours } \\
\text { intellectuel } \\
\text { conformisation } \\
\text { contrainte }\end{array}$ & 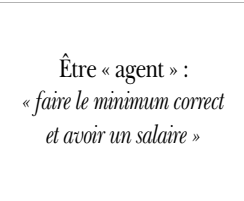 \\
\hline
\end{tabular}

\subsubsection{Gérer les tensions entre image de soi pour soi et de soi pour autrui : une reconnaissance extérieure}

L'implication au sein du club d'escrime permet à Max de construire une image de soi pour soi et de soi pour autrui très valorisante. Créateur de cette structure, il se sent «investi à la fois d'une mission de père de famille et de chef d'entreprise ». Si les multiples fonctions qu'il est amené à assumer lui donnent aussi une polyvalence de compétences dont il est fier, en revanche, cet engagement associatif semble très critiqué par ses (ex-) collègues du collège, sur les propos desquels il revient sans cesse, alors qu'il travaille désormais dans un autre établissement. On sent un fort ressentiment face à ceux 
qui le prenaient " pour un fumiste », qualificatif qu'il récuse. Inspecté peu de temps avant l'enquête et remis en cause au niveau de ses pratiques pédagogiques à cette occasion, il renvoie l'ensemble de ses adversaires - et la formation continue - du côté des discours et d'une EPS à laquelle il ne croit plus. On retrouve donc, à titre de défense, les oppositions citées plus haut. Mais cette situation conflictuelle reste difficile à tenir. Il lui faut trouver une forme de cohérence interne, justifiant de ses choix et permettant la réorganisation de l'ensemble de ses actions.

\subsubsection{Dépasser les clivages à travers un rôle d'éducateur : entre diversité et unité}

Pour conserver un équilibre, au-delà de la critique faite à ceux dont il ne partage pas (plus) les conceptions, il se situe comme un éducateur et cela donne sens à sa vie. Face à des élèves «qui sont tous à rééduquer... qui manquent de structures", il peut s'investir: le public de ZEP entre facilement dans cette perspective éducative prioritaire. Les licenciés du club, d'origines sociales très diversifiées, ont « besoin d'être encadrés », même si par ailleurs, il leur reproche un manque d'autonomie. Enfin, le projet d'adoption de deux enfants de la DASS va aussi dans ce sens et réorganise l'ensemble des actions entreprises autour d'une valeur suprême : éduquer. Conscient que les besoins des lycéens s'avèrent moins importants sur ce registre, il dépasse ce constat en donnant peu de place à l'EPS et aux enseignants qui la font, ce qui lui permet de prendre un mi-temps sans trop de regrets, relativisant son rôle d'éducateur dans ce nouveau contexte.

\subsubsection{Le monde de Michèle}

\subsubsection{Un rapport relativement harmonieux entre continuité et changement}

Fille d'instituteurs, mariée à un instituteur, Michèle voit, à travers ses enfants (l'un sera professeur des écoles, l'autre enseignant d'EPS), se perpétuer le métier d'enseignant et le partage d'une culture commune.

Mais dans son cas, si la peur d'échouer lui a fait prendre des chemins de traverse, si elle a commencé à enseigner en maternelle, sans formation, le désir d'être professeur d'EPS restait intact. Réaliser, à 42 ans, un rêve formulé dès le collège, s'accompagne d'un sentiment d'accomplissement. L'institution lui a permis de changer progressivement de statut, puis d'oser tenter un concours, de le réussir et d'être intégrée dans un groupe professionnel qu'elle aspirait à rejoindre.

Quoi qu'il en soit, son expérience passée en primaire reste fondatrice d'une identité en mutation. Pourtant, on voit apparaitre, dans le discours de Michèle certaines tensions entre une discipline éducative, permettant la « réussite de tous les élèves ", valorisant les processus d'apprentissage (proche de l'éducation en primaire), et une vision qu'elle juge plus « rétrécie » de l'EPS, partagée par de nombreux collègues avec lesquels elle est en désaccord, centrée sur l'enseignement des APSA. Cette position ne correspond pas aux valeurs qu'elle défend mais rencontre l'adhésion d'un certain nombre d'élèves qui entrent en résistance avec son approche jugée trop peu conforme aux images sportives véhiculées par les médias.

Tableau 2. Structure des oppositions dans le discours de Michèle

\begin{tabular}{|l|l|}
\hline \multicolumn{1}{|c|}{$\begin{array}{c}\text { Vision de l'EPS dans l'enseignement } \\
\text { primaire (+) }\end{array}$} & \multicolumn{1}{|c|}{$\begin{array}{c}\text { Vision de l'EPS dans l'enseignement } \\
\text { secondaire (-) }\end{array}$} \\
\hline Éduquer et enseigner des savoirs fondamentaux & $\begin{array}{l}\text { Se centrer sur les APS comme pratique sociale de référence } \\
\text { et enseigner des savoirs spécifiques }\end{array}$ \\
\hline Réussite de tous les élèves & Réussite des meilleurs \\
\hline Évaluation formative (centrée sur les processus d'apprentissage) & Évaluation sommative (centrée sur les résultats) \\
\hline
\end{tabular}


Malgré tout, le contexte professionnel local dans lequel elle est reconnue pour ses compétences tant professionnelles que personnelles lui permet d'assumer son rôle d'« enseignanteéducatrice », et de mener à bien la mission qui lui a été confiée. Très investie dans un établissement rural qui a accompagné l'infléchissement de son parcours, porteuse de projets interdisciplinaires, elle coordonne et dynamise par ailleurs l'équipe disciplinaire : «On est trois, on se connaît depuis vingt ans. On travaille en commun. C'est un groupe qui fonctionne bien. » Cet ensemble d'éléments donne à son action une cohérence forte. Pourtant, dans ce nouvel univers, restent des enseignants d'EPS dans lesquels Michèle ne se reconnaît pas: ceux qui sont «globalement trop laxistes et ont oublié que leur métier consiste à enseigner et à donner aux élèves les moyens de progresser »; ceux qui, trop centrés sur la performance, oublient que tous les élèves n'ont pas les mêmes ressources; ceux enfin qui, trop focalisés sur leur discipline, jugent sévèrement les élèves sans tenir compte du contexte social dans lequel ils évoluent. Ces positions entrent doublement en conflit avec, d'une part, un parcours professionnel où la prise en compte de l'enfant dans sa globalité, à l'école primaire, constituait une évidence; d'autre part, un manque de formation initiale conduisant à privilégier des aspects plus transversaux dans l'enseignement des APSA.

3.2.2.2. La promotion interne au cour du rapport entre image de soi pour soi et pour autrui

"Promue de l'intérieur» (Dubar, 1991), valorisée par l'inspecteur lors de sa titularisation, Michèle est reconnue pour son engagement à l'intérieur comme à l'extérieur du collège. Impliquée dans différents projets, elle conçoit l'établissement comme une véritable communauté éducative. Son parcours lui a permis de prendre confiance et de développer une image relativement positive d'elle-même.

Pourtant, ce monde de l'EPS qu'elle a côtoyé de plus ou moins loin avant d'y entrer définitivement est loin d'être unanime sur la place à accorder à ceux dont les compétences ont été acquises de façon peu orthodoxe. Si des enseignants l'ont aidée dans son parcours professionnel, d'autres n'ont pas hésité à émettre certaines réserves. Elle exprime son désaccord face à ceux qui «critiquent l'inspecteur parce qu'il les pousse à travailler ", ceux qui remettent en cause la promotion des enseignants sans Licence et obtenant le CAPEPS interne; en un mot : « des gens avec qui il a fallu s'affronter sur le plan des personnes pour se faire reconnaître ». Car Michèle est entrée "par la petite porte » (Merton, 1957), dévalorisée ou jalousée par certains collègues. Là encore, un contexte professionnel favorable lui permet de vivre sa promotion interne dans une relative sérénité.

\subsubsection{Entre diversité et unité : une quête du savoir}

Investie à titre personnel dans des pratiques sportives qui participent de son équilibre, Michèle développe un large registre d'actions, en réseau et à différents niveaux: celui de l'élève, de la classe, de l'équipe EPS, de la discipline, de l'établissement, de la commune. Ses centres d'intérêt montrent aussi une réelle curiosité face à la connaissance: une soif de savoir qu'elle relie à son manque de formation initiale. Au-delà d'une participation assidue en formation continue EPS, elle a suivi après sa réussite au CAPEPS, un cursus en Sciences de l'Éducation qu'elle envisage de prolonger "pour éclairer la pratique pédagogique et éducative ", pour donner sens à son action au quotidien, dans un métier qu'elle a choisi et dans lequel elle se réalise pleinement.

\section{DISCUSSION}

Les résultats mettent en relief la diversité avec laquelle chaque enseignant, porteur d'une histoire singulière, investit son métier et se saisit des opportunités professionnelles ou personnelles qu'il rencontre. L'obtention du CAPEPS interne peut être considérée comme une voie 
de promotion professionnelle et de reconnaissance sociale mais l'entrée dans le métier, par défaut pour Max (qui doit délaisser l'escrime), par vocation pour Michèle (ce qui occasionne un détour bénéfique), joue un rôle déterminant par rapport à l'engagement dans la carrière.

Ainsi, on remarque que l'obtention d'un concours de recrutement de plus haut niveau ne produit pas toujours les effets escomptés par l'institution et que les trajectoires professionnelles s'organisent entre sentiment de compétence et espaces de reconnaissance sociale investis. Les espaces de reconnaissance (le club pour Max ; l'établissement pour Michèle) participent de la construction identitaire et invitent le sujet à s'investir davantage. Pour Max, le changement de statut s'accompagne d'une reconnaissance institutionnelle qu'il minimise car l'État ne fait que réparer certaines injustices : «c'est pas normal que des enseignants qui font le même travail aient un salaire inférieur et des horaires plus lourds ». Par ailleurs, il juge ses compétences «supérieures à celle d'un simple professeur d'EPS » car associées à des registres plus larges développés dans le club. Ces positions entrent en conflit avec la non-reconnaissance de l'équipe EPS du collège qui lui reproche son manque de travail lié à une forte implication à l'extérieur de l'établissement. Max tente donc de «faire la preuve » de sa valeur ailleurs (dans le club), et dans un autre établissement (lycée) où l'EPS est peu valorisée et l'équipe plus tolérante par rapport à son faible investissement. Pour Michèle, la reconnaissance existe déjà au sein et hors de l'établissement. La réussite du concours représente une victoire sur elle-même et lui a permis de se dépasser, de se rassurer sur ses compétences professionnelles construites sans formation initiale spécifique.

De ce point de vue, l'analyse permet de repérer le poids du parcours scolaire, de la formation initiale et de l'histoire sportive dans la relation des enseignants aux savoirs théoriques et pratiques. Représentations du métier et de la discipline sous-tendent et justifient des choix d'implication en interne ou en externe par rapport au système scolaire. Le rapport à la formation professionnelle (Altet, 2000) comme moyen de promotion (sociale ou personnelle) est sensiblement différent chez les deux enseignants. Il est «instrumental» chez Max pour qui passer le CAPEPS permet d'obtenir un congé et de mieux s'occuper du club. Pour souligner le peu de crédit accordé à cette opération de promotion, il défend l'idée que l'utilité ou l'efficacité sur le terrain se mesure à l'apprentissage de tours de mains, la mise en œuvre de recettes ayant fait leurs preuves. Critique envers des formations qu'il juge trop théoriques, il refuse désormais d'y participer.

En revanche, ce rapport à la formation est «professionnel » chez Michèle: cette enseignante s'inscrit dans un processus de construction progressive de compétences professionnelles, à la recherche d'articulations théorie-pratique, individu-groupe (équipe EPS), textes nationaux - contexte local, etc. Après un début de carrière directement sur le terrain pédagogique, elle obtient au bout de quelques années un CAP d'institutrice. Cette enseignante ne s'inscrit pourtant pas dans ce clivage théorie-pratique de la même manière et affiche une réelle forme de reconnaissance envers l'institution qui lui a permis de réaliser son rêve. Au-delà de sa forte motivation pour enseigner l'EPS, son chemin l'a conduite à enseigner le français; cette expérience lui donne certaines compétences vis-à-vis de l'écrit et « un temps d'avance » par rapport aux problématiques qui traversent l'école aujourd'hui et face auxquelles de nombreux enseignants d'EPS se trouvent démunis : elle peut s'inscrire avec aisance dans les projets mis en place au sein du collège (parcours croisés, parcours diversifiés, itinéraires de découverte, etc.). En revanche, un autre problème se pose pour elle, celui de la césure entre « vrais » et « faux » professionnels (Merton, 1957). Michèle le dépasse par un investissement irréprochable, une recherche de mise en cohérence des actions au 
niveau de la classe, de l'équipe et de l'établissement, répondant ainsi aux exigences du texte sur la Mission des Enseignants (1997). Convaincue de ses manques en termes de formation initiale spécifique et de la nécessité de questionner sa pratique, elle continue à se former.

Enfin, le développement professionnel reste très lié au contexte institutionnel et humain. L'évolution de la discipline a engendré des prises de positions relativement contrastées au sein du groupe professionnel. La dimension de l'équipe semble très importante pour les enseignants d'EPS. L'engagement professionnel se décline différemment selon que le contexte humain est jugé favorable ou défavorable. Chacun va donc utiliser, dans la mesure du possible, des stratégies d'adaptation. Par exemple, Max quitte son collège dans lequel l'équipe le remet en cause pour son manque d'investissement et prend un mi-temps au lycée, ce qui lui donne un motif valable pour se mettre à distance; cette situation est rendue possible parce que ses nouveaux collègues semblent moins préoccupés de cohérence et d'innovation que les précédents.

Michèle enseigne depuis plus de vingt ans dans le même collège rural et peut reproduire, à échelle humaine, le suivi des élèves à l'image de ce qui est demandé à un enseignant du primaire. Son itinéraire professionnel, riche d'expériences diversifiées, l'a amenée à s'inscrire dans une dynamique dont le «petit monde » du collège bénéficie largement. Son but : renforcer les liens, fédérer et dynamiser.

\section{CONCLUSION}

Au terme de cette étude, on remarque qu'au-delà de ces arrangements avec soi-même, les autres et le contexte, certains processus se font jour dans les dynamiques identitaires. En fait, les identités «bricolées » entre soi et l'environnement conduisent à la « construction d'un monde » cohérent pour le sujet, qu'il justifie en prenant appui sur des représentations profes- sionnelles, des valeurs en lien avec les pratiques. Pour Dubar (1998), l'identité des individus est sans cesse réajustée et renégociée. Cette construction, qui se traduit par des formes identitaires distinctes, s'opère au moyen d'une interaction permanente entre les représentations de soi et le regard d'autrui. Mais cette " organisation » reste toujours provisoire et invite à des études longitudinales pour repérer dans quelle mesure la modification d'éléments personnels ou d'éléments liés au contexte peuvent amener les enseignants à recomposer, au moins en partie, leur univers professionnel au cours de la carrière (RouxPerez, 2005).

\section{BIBLIOGRAPHIE}

Altet, M. (2000). « L'analyse de pratiques, une démarche de formation professionnalisante? ", Recherche et Formation, 35, 25-41.

Ardoino, J. (1992). "L'approche multiréférentielle des situations éducatives et formatives", Pratiques de formation : Analyses, 25-26, Université de Paris VIII.

Barbier, J.-M. (1996). " De l'usage de la notion d'identité en recherche ", Éducation permanente, $\mathrm{n}^{\circ}$ 128, p. 11-26.

Charlot, B. (1997). Du rapport au savoir, Paris, Anthropos.

Demazière, D. et Dubar, C. (1997). Analyser les entretiens biographiques, Paris, Nathan.

Derouet, J.-L. (1992). École et justice, Paris, Métailié.

DUBAR, C. (1991). La socialisation: construction des identités sociales et professionnelles, Paris, Armand Colin.

Dubar, C. (1992). "Formes identitaires et socialisation professionnelle ", Revue Française de Sociologie, $\mathrm{n}^{\circ} 4$, p. 505-529.

Dubar, C. (1998). «Socialisation et construction identitaire ", in J.-C. Ruano-Borbalan (éd.), L'identité : l'individu, le groupe, la société, Auxerre, Sciences humaines, p. 135-141.

DuBar, C. (2000). La crise des identités, linterprétation d'une mutation, Paris, PUF.

GHIGLIONE, R. (1991). « L'analyse propositionnelle du discours dans tous ses états", in R. Ghiglione et A. Blanchet (éds), Analyse de contenu et contenus d'analyses, Paris, Dunod.

Huberman, M. (1989). La vie des enseignants : évolution et bilan d'une profession, Neuchâtel, Delachaux-Niestlé.

Hughes, E. C. (1937). "Institutional office and the person", American Journal of Sociology, $\mathrm{n}^{\circ} 43,3$, p. 404-413. 
Ion, J. (1990). Le travail social à l'épreuve du territoire, Toulouse, Privat.

Jodelet, D. (1989). Les représentations sociales, Paris, PUF.

KAdDOURI, M. (2000). « Retour réflexif sur les dynamiques identitaires ", in C. Gohier et C. Alin (éds), Enseignant-Formateur : la construction de l'identité professionnelle, Paris, L'Harmattan, p. 195-212.

LAHIRE, B. (1998). L'homme pluriel, Paris, Nathan.

Lautier, N. (2001). Psychosociologie de l'éducation, Paris, Arnsmand Colin.

MerTon, R. (1957). Social Theory and Social Structure, New York, The Free Press.

MIAS, C. (1998). L'implication professionnelle dans le travail social, Paris, L'Harmattan.

Moscovici, S. (1961). La psychanalyse, son image et son public, Paris, PUF.
RouX-Perez, T. (2001). Des processus de construction de l'identité professionnelle des enseignants d'Éducation Physique et Sportive : entre appartenance au groupe, expériences professionnelles singulières et recompositions identitaires à l'échelle du temps, Thèse de doctorat non publiée, Université de Nantes.

RouX-PEREz, T. (2003). «Identité professionnelle et modes d'implication privilégiés chez les enseignants d'EPS ", Les Sciences de l'Éducation pour l'Ère Nouvelle, n 36, 4, p. 37-68.

RouX-PEREZ, T. (2005). " Dynamiques identitaires à l'échelle du temps : une étude de cas chez les enseignants d'Éducation Physique et Sportive ", Science et Motricité, $n^{\circ} 56$, p. $75-96$.

TAP, P. (1998). « Marquer sa différence », in J.-C. RuanoBorbalan (éd.), L'identité : l'individu, le groupe, la société, Auxerre, Sciences humaines, p. 65-68.

\section{ZusammENFAsSung : Identitätsprozesse in der Lehrerkarriere: zwei Fallstudien zum Sportunterricht}

In der Studie geht es um die Karriere von Sportlehrern, die Art wie sie ihre professionelle Laufbahn einschätzen, dort Fixpunkte finden und Strategien entwickeln, um dem Kontext ihrer Arbeit einen Sinn zu geben. Sie legt den Schwerpunkt auf die Aneignung von Bildungsangeboten und ihren Einfluss auf eventuelle Transformationsprozesse hinsichtlich der professionellen Identität. Eine schriftliche Befragung von 381 Probanden wurde durch 12 semi-direktive Interviews ergänzt. Als Kriterium für die Auswahl der zwei Fallstudien diente die kurze oder nicht vorhandene Sportlehrerausbildung. Beide wurden im Laufe ihrer Karriere aufgrund eines Auswahlverfahrens befördert. Die Resultate zeigen die Vielfalt mit der jeder Lehrer mit seiner jeweils eigenen Geschichte sich in seinen Beruf einbringt und die beruflichen oder persönlichen Gelegenheiten nutzt, die sich bieten. Wir stellen fest, dass der Erwerb des höchsten Diploms nicht immer die von der Institution intendierten Effekte mit sich bringt. Die Organisation der beruflichen Laufbahnen ist abhängig vom Gefühl, der Kompetenz und den Räumen der sozialen Anerkennung.

SCHLAGWÖRTER : berufliche Identität, Karriere, Repräsentationen, Ausbildung.

\section{RIassunto : Processi identitari nella carriera degli insegnanti: due studi del caso in Educazione Fisica e Sportiva (EFS)}

Lo studio si interessa alla carriera degli insegnanti di EFS reperendo la maniera con cui strutturano il loro percorso professionale, trovano degli ancoraggi e sviluppano delle strategie per dare del senso al loro contesto di lavoro. Esso si collega alle modalità di appropriazione dei dispositivi di formazione e al loro impatto su eventuali processi di trasformazione in termini d'identità professionale.

Una inchiesta con questionario su 381 soggetti è stata completata da interviste semidirettive per 12 di loro. I due insegnanti presentati in quest'articolo sono stati scelti sulla base di un'entrata nel mestiere facente seguito a studi molto brevi, perfino inesistenti nel settore dell'EFS. L'uno e l'altro hanno superato, nel corso della carriera, un concorso di reclutamento che gli ha permesso di accedere ad uno statuto superiore.

I risultati mettono in rilievo la diversità con la quale ciascun insegnante, portatore di una storia singolare, investe il suo mestiere e si prende delle opportunità professionali o personali che incontra. Si osserva che l'ottenimento di un diploma del più alto livello non produce sempre gli effetti attesi dall'istituzione. Le 
traiettorie professionali si organizzano tra sentimento di competenza e spazi di riconoscimento sociale investito.

PAROLE CHIAVE : carriera, formazione, identità professionale, rappresentazioni.

\section{REsumen : Procesos identitarios en la carrera de los docentes : dos estudios de casos en educación fisica y deportiva}

El estudio se interesa por la carrera de los profesores de EPS poniendo de relieve la manera en que comprenden su trayectoria profesional, encuentran en ello anclaje y desarrollan estrategias para dar sentido a su contexto de trabajo. Se dedica a las modalidades de apropiación de los dispositivos de formación y a su impacto en posibles procesos de transformación en término de identidad profesional. Una investigación por cuestionario con 381 temas fue completada por conversaciones semidirectivas para 12 de ellos. Se eligió a los dos profesores presentados en este artículo sobre la base de una entrada en la profesión que se inscribia a continuación de estudios muy cortos o incluso inexistentes en el ámbito de la edución fisica. Uno y otro consiguieron oposiciones, durante su carrera, para acceder a un estatuto superior. Los resultados ponen de relieve la diversidad con la cual cada profesor, con su historia singular, invierte su profesión y se agarra de las oportunidades profesionales o personales que encuentra. Se observa que la obtención de un título de nivel más alto no produce siempre los efectos esperados por la institución. Las trayectorias profesionales se organizan entre sentimiento de competencia y espacios de reconocimiento social invertidos.

PALABRAS ClaVES : identitad profesional, carrera, representaciones, formación. 